\title{
Parâmetros Genéticos do Peso no Início da Estação de Monta, Considerado Indicativo do Peso Adulto de Matrizes Nelore ${ }^{1}$
}

\section{Maria Eugênia Zerlotti Mercadante ${ }^{2}$, Alexander George Razook ${ }^{1,3}$, José Benedito de Freitas Trovo $^{4}$, Joslaine Noely dos Santos Gonçalves Cyrillo ${ }^{2}$, Leopoldo Andrade de Figueiredo ${ }^{2}$}

RESUMO - Parâmetros genéticos foram estimados para peso na entrada da monta (PEM), considerado como indicativo do peso adulto de vacas Nelore. O arquivo de dados continha 7.902 registros de 1.556 vacas pertencentes a um experimento de seleção conduzido na Estação Experimental de Zootecnia de Sertãozinho, SP, Brasil. O PEM foi analisado como o último peso disponível para cada vaca no arquivo (PEM_U) ou como registros repetidos, incluindo todos os pesos (PEM_R). As análises foram feitas também em dois outros arquivos excluindo os registros das vacas descartadas antes de chegar aos 4 anos de idade, para o último peso registrado (PEM_U2) e para os registros repetidos (PEM_R2). Os componentes de variância foram estimados por máxima verossimilhança restrita, ajustando modelo animal uni e multivariado. O modelo multivariado incluiu pesos à seleção ajustados para 378 (somente machos) e 550 (somente fêmeas) dias de idade. As estimativas de herdabilidade obtidas nas análises univariadas foram $0,30 \pm 0,05 ; 0,37 \pm 0,06$; $0,35 \pm 0,04$; e 0,42 $\pm 0,05$, para PEM_U, PEM_U2, PEM_R e PEM_R2, respectivamente. Os valores correspondentes para as análises multivariadas

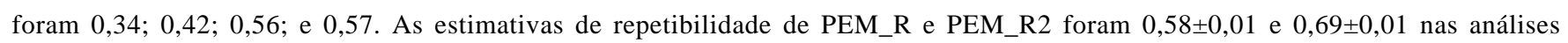
univariadas e 0,61 e 0,72 nas análises multivariadas. As estimativas da mudança genética em PEM foram positivas e significativas, iguais

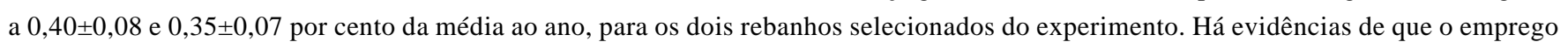
de modelos multivariados incluindo registros repetidos e os pesos à seleção de machos e fêmeas seja mais apropriado que a utilização de apenas o último PEM como indicativo do peso adulto. O PEM poderia ser incluído em um programa de seleção visando mudanças (aumento ou diminuição) ou monitoração, para manter o peso adulto desejável.

Palavras-chave: bovinos de corte, crescimento, correlação genética, herdabilidade, repetibilidade

\section{Genetic Parameters for Weight at the Beginning of Breeding Season, Considered Indicative of Mature Weight of Nelore Cows}

\begin{abstract}
Genetic parameters were estimated for weight at the beginning of breeding season (PEM), considered as an indicative of mature weight of Nelore cows. Data file comprised 7,902 records from 1,556 cows from to a selection experiment conducted at the Estação Experimental de Zootecnia de Sertãozinho, SP, Brazil. PEM were analyzed either as the last weight available for each cow in the data file (PEM_U) or as repeated records, including all weights (PEM_R). The analyses were also performed excluding the records of cows culled before reaching 4 years of age, and for both the last (PEM_U2) and the repeated (PEM_R2) records. Variance components were estimated by restricted maximum likelihood, fitting single and multiple trait animal models. The multiple trait models included the selection weights adjusted for 378 (males only) and 550 (females only) days of age. Heritability estimates obtained from the single trait analyses were $0.30 \pm 0.05,0.37 \pm 0.06,0.35 \pm 0.04$ and $0.42 \pm 0.05$, for PEM_U, PEM_U2, PEM_R and PEM_R2, respectively. Corresponding values for the multiple trait analyses were $0.34,0.42,0.56$, and 0.57 . Repeatability estimates for PEM_R and PEM_R2 were $0.58 \pm 0.01$ and $0.69 \pm 0.01$ for the single, and 0.61 and 0.72 for the multiple trait analyses, respectively. Estimates of genetic changes were significantly positive and equal to $0.40 \pm 0.08$ and $0.35 \pm 0.07$ percent of the mean per year, for the two selected lines in the experiment. The results obtained in the present study indicated the multiple trait repeated records models as the most appropriate for analyzing the PEM as an indicative of mature weight. The PEM could be included in a selection program aiming at monitoring a desirable mature weight.
\end{abstract}

Key Words: beef cattle, growth, genetic correlation, heritability, repeatability

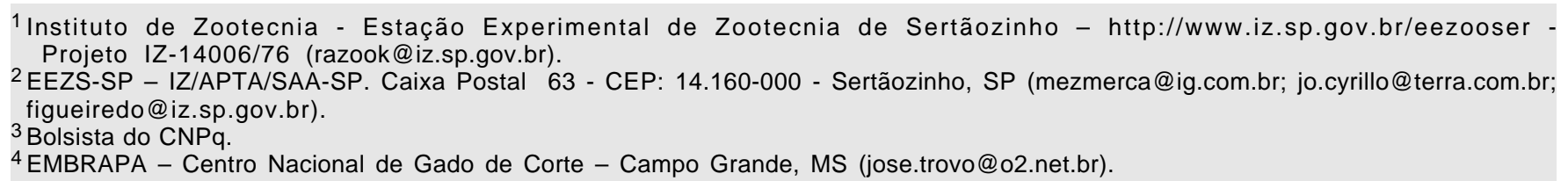




\section{Introdução}

Uma das considerações chaves na avaliação das consequências de maior crescimento é relacionar o impacto no rebanho de matrizes (Johnston et al., 1996). Mas como definir peso adulto com a finalidade de incluí-lo em uma avaliação genética? Segundo Bullock et al. (1993), há vários procedimentos pelos quais o peso adulto pode ser estimado, e o mais óbvio deles seria uma média de todos os pesos tomados após o animal ter parado de crescer. Este procedimento fornece um acesso acurado ao peso adulto, entretanto limita muito o número de animais que podem ter seus registros utilizados. Estudos sobre curva de crescimento de fêmeas zebuínas têm mostrado que a idade à maturidade, momento em que os animais atingem $95 \%$ do peso assintótico, está entre 3,7 e 5,3 anos (Oliveira et al., 2000; Garnero, 2002), entretanto, a não inclusão de fêmeas que foram descartadas no início da vida reprodutiva (2 e 3 anos de idade) faz com que esta amostra seja ainda menor e mais selecionada. Análises univariadas pressupõem que o descarte dos animais foi aleatório, enquanto análises multivariadas levam em conta o descarte não-aleatório, explorando as correlações genéticas e ambientais entre uma medida tomada antes da seleção dos animais e pesos adultos perdidos subseqüentemente (Meyer, 1995; Kaps et al., 1999); dessa forma, estas últimas são alternativas para avaliação genética do peso adulto. Johnston et al. (1996) sugeriram mais estudos de peso adulto com outros caracteres para permitir sua inclusão em uma análise multivariada completa.

Entre os trabalhos relatando parâmetros genéticos para peso adulto, alguns autores utilizaram um único peso a partir dos 4 anos de idade (Silva et al., 2000; Rosa et al., 2001), outros analisaram medidas repetidas, considerando pesos a partir dos 5 anos (Kaps et al., 1999) ou, todos os pesos disponíveis (Arango \& Plasse, 2002; Choy et al., 2002), permitindo incluir todas as fêmeas que entraram na monta, e outros ainda usaram o parâmetro $\mathbf{A}$ estimado a partir de curva não-linear de crescimento (Meyer, 1995, entre outros). As vantagens do uso de medidas repetidas em contraste com medida única, como maiores acurácia e possibilidade de ajuste dos efeitos ambientais, já são bem conhecidas. Nestes trabalhos, como era de se esperar, as herdabilidades estimadas variaram bastante, sendo iguais a 0,38 para Canchim (Silva et al., 2000), de 0,26 a 0,36 para Nelore (Rosa et al., 2000 e 2001), de 0,44 a 0,53 para Angus (Kaps et al., 1999), de 0,49 $\pm 0,10$ a $0,68 \pm 0,07$ para Brahman (Arango \& Plasse, 2002) e de 0,47 a 0,73 para Hereford e cruzados (Meyer, 1995). Os maiores valores foram provenientes de análise de medidas repetidas de vários pesos ou do parâmetro $\mathbf{A}$ de curvas não-lineares de crescimento. Lôbo et al. (2000) relataram valor de herdabilidade de $0,28 \pm 0,05$, média de cinco estudos com bovinos criados em países tropicais, enquanto Koots et al. (1994) observaram valor bem superior $(0,50 \pm 0,02)$, média de 25 estudos com bovinos criados, em sua maioria, em países de clima temperado.

O objetivo deste estudo foi estimar e comparar parâmetros genéticos para peso adulto de fêmeas usando modelo animal em análise univariada sem medidas repetidas, com medidas repetidas, incluindo ou não registros das fêmeas de 2 e 3 anos de idade, e modelo animal em análise trivariada com registros de machos e fêmeas antes da seleção e peso adulto das fêmeas, com a finalidade de discutir as várias opções de inclusão do peso adulto em uma avaliação genética.

\section{Material e Métodos}

As informações são referentes aos três rebanhos da raça Nelore do Experimento de Seleção da Estação Experimental de Zootecnia de Sertãozinho, Nelore Controle $(\mathrm{NeC})$, Nelore Seleção $(\mathrm{NeS})$ e Nelore Tradicional (NeT). Os machos foram selecionados segundo diferencial de seleção máximo (NeS e NeT) e nulo $(\mathrm{NeC})$, dentro de rebanho e ano, do peso padronizado aos 378 dias de idade (P378), e as fêmeas, segundo diferencial de seleção máximo (NeS e NeT) e nulo $(\mathrm{NeC})$ do peso padronizado aos 550 dias de idade (P550), mas em geral foram retidas anualmente $50 \%$ delas. O NeT, em um esquema mais flexível, recebeu touros de outros rebanhos, assim como, eventualmente, alguns touros e matrizes descartados dos $\mathrm{NeC}$ e NeS. Os detalhes da implantação dos rebanhos e do processo seletivo foram descritos por Razook et al. (1998) e Mercadante et al. (2003a).

Foram analisados os registros do peso à seleção, P378 dos machos e P550 das fêmeas, nascidos de 1978 a 2001, e dos pesos das fêmeas obtidos anualmente em novembro (no início do período das águas) na entrada da estação de monta (PEM), utilizado como indicativo do peso adulto. Nas matrizes que ainda estavam prenhes no início da estação de monta (aproximadamente 5\% delas), o PEM foi tomado após o parto, quando efetivamente entraram

\section{R. Bras. Zootec., v.33, n.5, p.1135-1144, 2004}


no lote de monta. Os registros de PEM são referentes a 22 anos de monta (1978 a 2000) de vacas nascidas de 1960 a 1998. Como as primeiras progênies dos rebanhos separados $\mathrm{NeC}, \mathrm{NeS}$ e NeT nasceram em 1981, dados de animais nascidos em anos anteriores foram identificados como rebanho original $(\mathrm{NeO})$. O peso na entrada da monta foi analisado como o último PEM da fêmea, incluindo (PEM_U) ou não(PEM_U2) os registros provenientes de fêmeas de 2 e 3 anos de idade, e como registros repetidos, do mesmo modo, incluindo (PEM_R) ou não (PEM_R2) os registros provenientes de fêmeas de 2 e 3 anos de idade.

A decisão de incluir (ou não) registros de fêmeas com 2 e 3 anos de idade foi suportada pela análise das médias ajustadas de altura na garupa tomadas no mesmo momento do PEM, mostrando que estas não se alteraram significativamente dos 4 anos em diante. Além disso, a escolha do último PEM da matriz e não o primeiro mais próximo aos 5 anos de idade, como sugerido por Silva et al. (2000) e Rosa et al. (2001), foi para possibilitar a inclusão das matrizes, já em idades mais avançadas, que iniciaram o experimento e que não tinham registros de PEM anteriores.

Para definição dos modelos quanto aos efeitos fixos, os caracteres foram analisados usando procedimento para modelos lineares mistos (PROC MIXED) do SAS (2000). Os efeitos fixos de interesse e suas interações foram considerados em todas as análises preliminares e, quando não-significativos $(\mathrm{P}>0,05)$, foram retirados do modelo. O modelo para os pesos à seleção, igual para P378 e P550, incluiu os efeitos fixos de grupo contemporâneo composto por ano de nascimento e rebanho (Tabela 1), mês de nascimento $(\mathrm{i}=8, \ldots, 11)$ e classe de idade da mãe $(i=3, \ldots, \geq 10$ anos). Para PEM_U, PEM_U2, PEM_R e PEM_R2, o modelo incluiu os efeitos fixos de grupo contemporâneo composto por ano de entrada na monta $(i=1978, \ldots, 2000)$ e rebanho (Tabela 1), do estado reprodutivo anterior $(\mathrm{i}=0$ : novilhas e vacas vazias, 1 : vacas prenhes) e da classe de idade da vaca na monta ( $\mathrm{i}=2, \ldots, \geq 9$ anos para PEM_U e PEM_R e $i=4, \ldots, \geq 9$ anos para PEM_U2 e PEM_R2). A estrutura geral dos dados utilizados é apresentada na Tabela 1.

Os componentes de variância foram estimados por máxima verossimilhança restrita usando o software MTDFREML (Boldman et al., 2002). Para P378 e P550, o modelo misto incluiu os efeitos fixos descritos acima, além dos efeitos aleatórios genético direto do animal e permanente de ambiente da mãe, para PEM_U e PEM_U2, somente o efeito aleatório genético direto do animal e, para PEM_R e PEM_R2, os efeitos aleatórios genético direto do animal e permanente de ambiente do animal. Os pesos à seleção foram incluídos somente nas análises trivariadas e os PEM foram estudados em análises uni e trivariadas. Em todas as análises, foi usada uma matriz de parentesco com 6.588 animais, cuja população base são pais dos animais que nasceram a partir de 1978.

O modelo geral é descrito a seguir:

$$
\mathbf{y}=\mathbf{X} \beta+\mathbf{Z}_{\mathbf{a}} \mathbf{a}+\mathbf{Z}_{\mathbf{p m}} \mathbf{p m}+\mathbf{Z}_{\mathbf{c}} \mathbf{c}+\mathbf{e}
$$

em que y é o vetor dos caracteres observados; $\beta$, um vetor de efeitos fixos; $\mathbf{a}$, um vetor de efeitos genéticos

Tabela 1 - Estrutura dos dados e estatísticas descritivas Table 1 - Data structure and descriptive statistics

\begin{tabular}{lccccccc}
\hline $\begin{array}{l}\text { Caracter } \\
\text { Trait }\end{array}$ & $\begin{array}{c}\text { Animais com registros } \\
\text { Animals with records }\end{array}$ & $\begin{array}{c}\text { Registros } \\
\text { Records }\end{array}$ & $\begin{array}{c}\text { Touros } \\
\text { Sires }\end{array}$ & $\begin{array}{c}\text { Mães } \\
\text { Dams }\end{array}$ & $\begin{array}{c}\text { GC } \\
\text { Média (kg) } \\
\text { Mean (kg) }\end{array}$ & $\begin{array}{c}\text { Desvio-padrão (kg) } \\
\text { Standard deviation }(\mathrm{kg})\end{array}$ \\
\hline P378 & 2658 & 2658 & 195 & 1075 & 66 & 306 & 37 \\
P550 & 2569 & 2564 & 184 & 1047 & 63 & 265 & 39 \\
PEM_U & 1556 & 1556 & 182 & 854 & 64 & 419 & 66 \\
PEM_U2 & 1203 & 1203 & 160 & 708 & 58 & 441 & 65 \\
PEM_R & 1556 & 7902 & 182 & 854 & 69 & 401 & 49 \\
PEM_R2 & 1203 & 5618 & 160 & 708 & 63 & 428 & \\
\hline
\end{tabular}

P378: peso padronizado aos 378 dias de idade (só machos); P550: peso padronizado aos 550 dias de idade (só fêmeas); PEM: peso na entrada da estação de monta; _U: considerando somente o último peso disponível para cada vaca; _U2: como _U, excluindo vacas descartadas dos rebanhos antes de 4 anos de idade; _R: considerando todos os registros disponíveis para cada vaca - medidas repetidas; R2: como $R$, excluindo vacas descartadas dos rebanhos antes de 4 anos e registros tomados antes de 4 anos de idade; GC: grupo contemporâneo (rebanho - ano de nascimento para P378 e P550, e rebanho-ano de entrada na monta para PEM).

P378: 378-day weight (males only); P550: 550-day weight (females only); PEM: weight at the beginning of breeding season; U: considering only the last record available for each cow;_U2: same as_U, excluding cows culled from the herds before 4 years of age; $R$ : considering all records available for each cow - repeated records; R2: same as_R, excluding cows culled from the herds before 4 years and records taken before 4 years of age; GC: contemporary group (herd-year of birth for P378 and P550, and herd-year of breeding season for PEM).

R. Bras. Zootec., v.33, n.5, p.1135-1144, 2004 
aditivos; pm, um vetor de efeitos de ambiente permanente materno; c, um vetor de efeitos de ambiente permanente do animal; e e, um vetor de efeitos residuais. $\mathbf{X}, \mathbf{Z a}, \mathbf{Z} \mathbf{p m}$ e $\mathbf{Z c}$ são matrizes de incidência relacionando $\beta, \mathbf{a}, \mathbf{p m}$ e $\mathbf{c}$ a $\mathbf{y}$. É assumido que $\mathrm{E}[\mathbf{y}]=\mathbf{X} \beta ; \operatorname{Var}(\mathrm{a})=\mathrm{A} \otimes \sum_{\mathrm{a}} \mathrm{e} \operatorname{Var}(\mathrm{e})=\mathrm{I} \otimes \sum_{\mathrm{e}}$, em que $\sum_{a}$ é a matriz de covariância genética aditiva; $\sum_{\mathrm{e}}$, a matriz de covariância residual; A, a matriz de parentesco, I, uma matriz identidade; e $\otimes$ denota produto direto entre matrizes. Para o efeito de ambiente permanente ajustado para as características P378 e P550, é assumido que $\operatorname{Var}(\mathrm{pm})=\mathrm{I} \otimes \sum_{\mathrm{pm}}$, em que $\sum_{\mathrm{pm}}$ é a matriz de covariâncias permanentes de ambiente materno e pm, o número de mães dos animais com registros; e para o efeito de ambiente permanente ajustado para a característica PEM, é assumido que $\operatorname{Var}(\mathrm{c})=\mathrm{I} \otimes \sum_{\mathrm{c}}$, em que $\sum_{\mathrm{c}}$ é a matriz de covariâncias permanentes de ambiente do animal e c, o número de animais com registros. Assume-se ainda que os vetores a, pm, c e e não sejam correlacionados entre si.

Os valores genéticos foram preditos após a convergência, quando a variância dos valores da função (-2logL) do simplex foi menor que $10^{-6}$. Com o objetivo de comparar as predições nas diferentes análises, foram computados coeficientes de correlação de Spearman entre os valores genéticos preditos para PEM nas quatro análises trivariadas (PEM_U, PEM_U2, PEM_R, PEM_R2).

A estimativa da tendência genética anual em cada rebanho experimental foi obtida por contrastes ortogonais, como mostrado por Johnson et al. (1999). Um vetor de coeficientes $(\mathrm{k})$ foi gerado e rodado no MTDFREML para produzir contrastes lineares dos valores genéticos ( $\hat{\mu})$ sobre os anos de nascimento (20 anos, 1981, ..., 2000). O vetor dos coeficientes $\mathrm{k}$ foi obtido a partir do elemento do vetor c'=-9,5: 1: 9,5, correspondente ao ano de nascimento do animal, dividido pelo número de observações do rebanho e ano de nascimento deste animal. Os coeficientes de regressão foram calculados como $k^{\prime} \hat{\mu} / c^{\prime} c$ e a variância de k'û no MTDFREML, como $\mathrm{V}(\mathrm{k} \hat{\mu})=\mathrm{k}^{\prime} \mathrm{L}^{22} \mathrm{k}$, em que $\mathrm{L}^{22}$ é a porção animal por animal da inversa da matriz dos coeficientes das equações de modelo misto na convergência dos componentes de variância. O erro-padrão dos coeficientes de regressão foi também obtido como $\left[\mathrm{V}\left(\mathrm{k}^{\prime} \hat{\mu}\right)\right]^{0,5} / \mathrm{c}^{\prime} \mathrm{c}$, e a significância das regressões foi determinada pelo teste $t$, com graus de liberdade igual ao número de anos no contraste menos 2.

\section{Resultados e Discussão}

As médias observadas do PEM, juntamente com o número de observações para cada idade, são mostradas na Figura 1. O número de observações diminui rapidamente com o aumento da idade, e o maior número de observações na classe de 12 anos, em relação às idades próximas, é decorrente da composição etária das vacas de $\mathrm{NeT}$ no início do experimento de seleção. Observa-se que as vacas continuaram a ganhar peso até 6-7 anos de idade. A média do peso das vacas de 5 anos ou mais, $437 \mathrm{~kg}$, mais baixa que a relatada na literatura para vacas Nelore adultas (Rosa et al., 2001), reflete o fato de o peso na entrada da monta ter sido tomado após o período seco, sempre menor que os registrados após o período das águas.

Os componentes de variância e parâmetros genéticos obtidos nas análises univariadas de PEM estão na Tabela 2. A inclusão do peso das vacas com 2 e 3 anos de idade na análise reduziu em ambos os casos (PEM_U e PEM_R) a estimativa da $\sigma_{\mathrm{a}}^{2} \mathrm{e}$, conseqüentemente, as análises sem estes registros forneceram estimativas de herdabilidade cerca de $20 \%$ maiores, porém com maior erro-padrão, em razão do menor número de animais. No caso das análises de medidas repetidas, a exclusão dos registros das vacas de 2 e 3 anos aumentou a estimativa de $\sigma_{c}{ }^{2}$ $\mathrm{e}$, conseqüentemente, de $\mathrm{c}^{2}$ e da repetibilidade $(\mathrm{t})$,

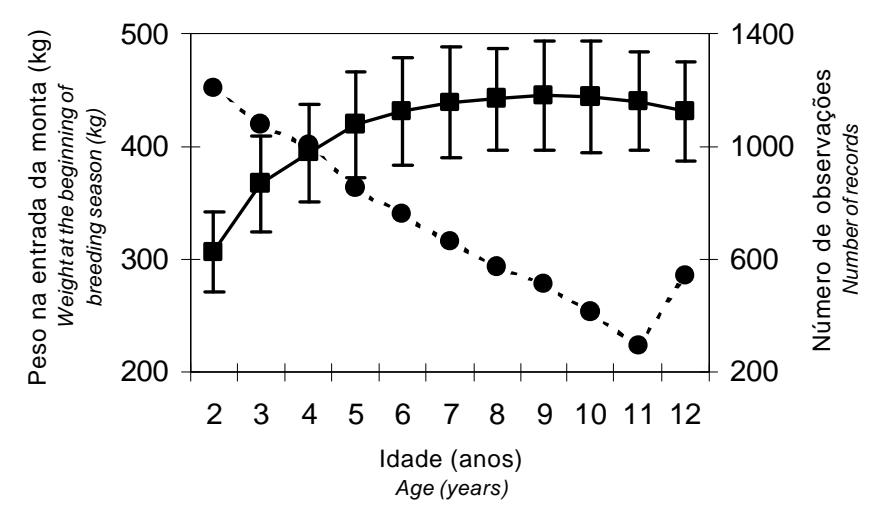

Figura 1 - Média (-), desvio-padrão e número de registros $(\bullet)$ observados para o peso na entrada da monta, por classe de idade.

Figure 1 - Observed mean ( $\mathbf{0})$, standard deviation and number of records $(\bullet)$ for the weight at the beginning of the breeding season by class of age. 
Tabela 2 - Componentes de variância e parâmetros genéticos do peso na entrada da estação de monta, estimados em análises univariadas

Table 2 - Variance components and genetic parameters of weight at the beginning of breeding season, obtained from single-trait analysis

\begin{tabular}{lcccccc}
\hline $\begin{array}{l}\text { Caracter } \\
\text { Trait }\end{array}$ & $\sigma_{\mathrm{a}}{ }^{2}\left(\mathrm{~kg}^{2}\right)$ & $\sigma_{\mathrm{c}}{ }^{2}\left(\mathrm{~kg}^{2}\right)$ & $\sigma_{\mathrm{e}}{ }^{2}\left(\mathrm{~kg}^{2}\right)$ & $\mathrm{h}^{2}$ & $\mathrm{c}^{2}$ & $\mathrm{t}$ \\
\hline PEM_U & 453 & & 1046 & $0,30 \pm 0,05$ & & \\
PEM_U2 & 621 & & 1048 & $0,37 \pm 0,06$ & & \\
PEM_R & 460 & 313 & 548 & $0,35 \pm 0,04$ & 0,24 & $0,58 \pm 0,01$ \\
PEM_R2 & 647 & 416 & 484 & $0,42 \pm 0,05$ & 0,27 & $0,69 \pm 0,01$ \\
\hline
\end{tabular}

PEM: peso na entrada da estação de monta; _U: considerando somente o último peso disponível para cada vaca; _U2: como _U, excluindo vacas descartadas dos rebanhos antes de 4 anos de idade; R: considerando todos os registros disponíveis para cada vaca - medidas repetidas; _R2: como_R, excluindo vacas descartadas dos rebanhos antes de 4 anos e registros tomados antes de 4 anos de idade; $\sigma_{a}{ }^{2}$, variância genética aditiva; $\sigma_{c}^{2}$, variância devida ao efeito permanente de ambiente; $\sigma_{e}{ }^{2}$, variância residual; $h^{2}$, herdabilidade, $c^{2}$, porção da variância total decorrente do efeito permanente de ambiente; $t$, repetibilidade.

PEM: weight at the beginning of breeding season; U: considering only the last record available for each cow; _U2: same as_U, excluding cows culled from the herds before 4 years of age; $R$ : considering all records available for each cow-repeated records; _R2: same as_R, excluding cows culled from the herds before 4 years and records taken before 4 years of age; $\sigma_{a}{ }^{2}$, additive genetic variance; $\sigma_{c}{ }^{2}$, permanent environmental variance; $\sigma_{e}{ }^{2}$, residual variance; $h^{2}$, heritability, $c^{2}$, proportion of phenotypic variance due to permanent environmental; $t$, repeatability.

aumentando a correlação entre os registros repetidos da mesma vaca. Análises preliminares excluindo registros das vacas abaixo de 5 anos (Mercadante et al., 2003b) mostraram valores de $\mathrm{c}^{2}$ e repetibilidade ainda maiores (cerca de $20 \%$ se comparados aos da análise de PEM_R2 do presente trabalho), porém com 50\% da amostra de vacas representadas em PEM_R (com todas as idades), uma vez que o maior descarte é observado entre 4 e 5 anos de idade (Mercadante et al., 2004). O fato de desconsiderar os registros das vacas de 2 e 3 anos de idade fez com que a amostra de animais com PEM ficasse cerca de $23 \%$ menor (Tabela 1). Um aspecto muito importante na modelagem de medidas repetidas é a pressuposição, quase sempre não muito válida em termos biológicos, de correlação genética igual a 1 entre medidas tomadas em diferentes idades.

Os padrões de comportamento dos componentes de variância sugerem que os registros do peso na entrada da monta provenientes das vacas com 2 e 3 anos de idade não podem ser considerados como peso adulto. Meyer (1995) observou tendência similar dos componentes de variância ao analisar registros de peso de vacas Hereford e cruzadas, incluindo ou não os registros daquelas com 3 anos de idade.

Com relação às análises de medidas repetidas, relativamente às análises de medida única, a $\sigma_{\mathrm{e}}{ }^{2}$ diminuiu cerca de $50 \%$ como era de se esperar, já que este componente é particionado em $\sigma_{\mathrm{c}}^{2}$ e $\sigma_{\mathrm{e}}^{2}$. As estimativas de herdabilidade foram mais altas, com erros-padrão menores, o que sugere uma análise muito mais acurada.

A magnitude do erro de predição e da acurácia dos valores genéticos obtidos, nas análises univariadas, somente daquelas vacas com registros em cada arquivo, assim como de seus pais, pode ser usada na comparação desses modelos. A distribuição dos valores de acurácia das quatro análises univariadas, para as vacas com registros (Figura 2), mostram que maiores acurácias dos valores genéticos podem ser obtidas quando os animais possuem registros repetidos (PEM_R e PEM_R2). Para as análises de PEM_U, PEM_U2, PEM_R e PEM_R2, as médias do erro de predição e de acurácia do valor genético para os touros, pais das vacas com registros, foram iguais a 16 e 0,$66 ; 19$ e 0,$66 ; 14$ e 0,75 ; e 17 e 0,73 , respectivamente, mostrando novamente a superioridade em acurácia das análises de medidas repetidas. Entretanto, a retirada dos registros das vacas de 2 e 3 anos de idade aumentou o erro de predição, reduzindo a acurácia, quando comparada à análise de medidas repetidas incluindo os registros das vacas jovens. Como a acurácia é função, além do erro de predição do valor genético, também da variância genética aditiva da característica em questão, maiores herdabilidades poderiam estar associadas sempre a maiores acurácias, entretanto, observando os resultados mostrados na Tabela 2, mesmo com variâncias genéticas aditivas semelhantes (PEM_U e PEM_R, por exemplo), obtêm-se acurácias cerca de $20 \%$ maiores nas análises considerando medidas repetidas. 


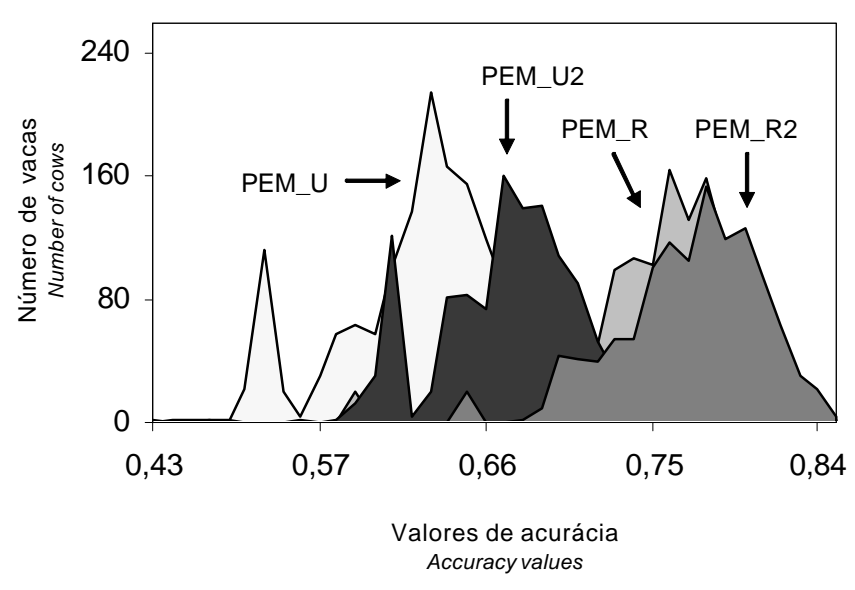

Figura 2 - Distribuição dos valores de acurácia associados aos valores genéticos das vacas com registros, obtidos em análises univariadas de PEM_U, PEM_U2, PEM_R e PEM_R2.

Figure 2 - Distribution of accuracy values associated to breeding values of cows with records, obtained from single-trait analysis of PEM_U, PEM_U2, PEM_R and PEM_R2.

Para conter o efeito do descarte das matrizes, o peso adulto deve ser considerado em uma análise multivariada juntamente com algum caracter correlacionado mensurado mais cedo na vida do animal e menos sujeito à seleção (Meyer, 1995; Kaps et al., 1999). Desse modo, análises trivariadas foram feitas com as quatro abordagens do PEM, juntamente com os pesos à seleção de machos $(\mathrm{P} 378)$ e de fêmeas (P550). As matrizes de variâncias e covariâncias completas dos efeitos genético-aditivo, permanente de ambiente materno (P378 e P550) e permanente do animal (PEM_R), e residual, além dos coeficientes de correlação, estimados somente nas análises trivariadas de PEM_R e PEM_R2, são mostrados na Tabela 3 . Os valores nulos da covariância entre os efeitos permanente de ambiente materno de P378 e P550 (classificados pela mãe) e permanente de ambiente de PEM_R ou PEM_R2 (classificados pelo animal), assim como da covariância entre os resíduos de P378 e P550 e de P378 e PEM_R ou PEM_R2 (animais diferentes) foram fixados de acordo com as pressuposições do modelo. Como observado nas análises univariadas, a inclusão dos registros das fêmeas com 2 e 3 anos de idade (análise de PEM_R) diminuiu substancialmente a estimativa da $\sigma_{\mathrm{c}}^{2}$ (Tabela 3) e do parâmetro $\mathrm{c}^{2}$, de 0,15 para 0,05 , reduzindo a repetibilidade de 0,72 para 0,61 (Tabela 4). Além disso, o comportamento de outros parâmetros, como as estimativas de herdabilidade dos P378 e P550, muito baixa para o primeiro $(0,28)$ e muito alta para segundo $(0,61)$, a correlação genética muito mais baixa entre eles $(0,59$, Tabela 3$)$ relativamente àquela estimada em análise bivariada destes dois pesos $(0,96$, resultados não mostrados) e a correlação muito alta entre P550 e PEM_R $(0,97$, Tabela 3), podem ser indícios de que este modelo realmente não é correto, devendo-se optar por não incluir os registros das vacas de 2 e 3 anos de idade em análises do peso adulto.

As correlações entre as classificações dos animais em função dos valores genéticos de PEM preditos nas quatro análises trivariadas, tanto para os touros dos rebanhos selecionados $\mathrm{NeS}$ e NeT como de todos os animais constantes na matriz de parentesco, são mostradas na Tabela 5. Os valores variaram entre 0,85 e 0,98 e foram maiores entre as soluções usando o último peso da matriz, incluindo ou não registros de 2 e 3 anos de idade.

A correlação entre as soluções obtidas nas análises de PEM_U2 e PEM_R2 foi alta $(0,95)$, sugerindo que, sem levar em conta o valor da herdabilidade, a análise do último peso da matriz sem os registros das fêmeas de 2 e 3 anos de idade poderia ser usada no caso de grandes bancos de dados ou no caso de recursos computacionais limitados. Entretanto, a acurácia dessas soluções nas análises trivariadas considerando somente o último registro da matriz é bem menor que aquelas considerando medidas repetidas de PEM. Como mostrado para as análises univariadas, as distribuições das acurácias do valor genético predito para as quatro abordagens do peso adulto em análises trivariadas, somente das vacas com registros, estão na Figura 3. Houve claramente ganho em acurácia, comparando análises com um só registro e com registros repetidos, e também comparando análises uni (Figura 2) e trivariadas. Entre estas últimas, o ganho em acurácia foi, em média, 8\% para PEM_U e PEM_U2 e 18\% para PEM_Re PEM_R2. Como observado nas análises univariadas, a retirada dos registros das vacas de $2 \mathrm{e}$ 3 anos de idade (PEM_U2 e PEM_R2) nem sempre é vantajoso nas análises de medidas repetidas, pois perde-se em acurácia.

As herdabilidades estimadas em modelos univariados (Tabela 2) para as quatro abordagens de peso adulto foram bem inferiores àquelas estimadas em modelos trivariados (Tabela 4). Meyer (1995) e Kaps et al. (1999) observaram aumento também substancial da herdabilidade dos pesos final e adulto,

\section{R. Bras. Zootec., v.33, n.5, p.1135-1144, 2004}


Tabela 3 - Componentes de covariâncias (da diagonal acima) e coeficientes de correlação (abaixo da diagonal) estimados em análises trivariadas

Table 3 - (Co)variance components (from the diagonals above) and correlation coefficient (below the diagonals) estimates obtained from multiple trait analyses

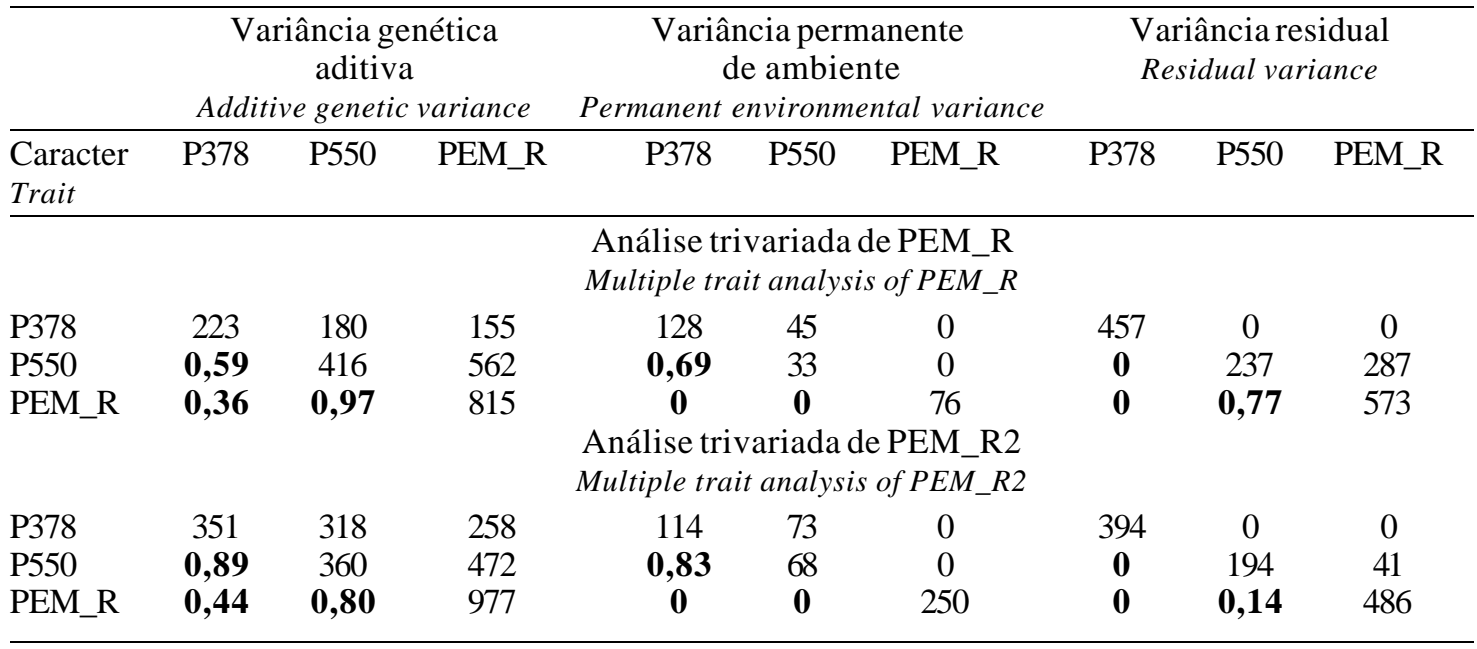

P378: peso padronizado aos 378 dias de idade (só machos); P550: peso padronizado aos 550 dias de idade (só fêmeas); PEM: peso na entrada da estação de monta; _R: considerando todos os registros disponíveis para cada vaca - medidas repetidas; R2: como_R, excluindo vacas descartadas dos rebanhos antes de 4 anos e registros tomados antes de 4 anos de idade.

P378: 378-day weight (males only); P550: 550-day weight (females only); PEM: weight at the beginning of breeding season; _R: considering all records available for each cow - repeated records; $R 2$ : same as_R, excluding cows culled from the herds before 4 years and records taken before 4 years of age.

respectivamente, quando medidas tomadas antes da seleção eram incluídas em análises multivariadas. Este aumento no componente de variância genético aditivo em análises multivariadas reflete a remoção do vício devido à seleção (Meyer, 1995). Nos rebanhos que geraram os dados analisados, a seleção foi praticada somente com base em um critério de seleção, P378 para machos e P550 para fêmeas; portanto, estas informações são essenciais para análise de caracteres obtidos após esta idade, principalmente daqueles correlacionados com os pesos à seleção, como é o caso do peso adulto. Em rebanhos em que há, por razões econômicas, uma pré-seleção ao desmame, e a seleção propriamente dita ao ano ou sobreano, estes caracteres poderiam ser analisados juntamente com o peso adulto para levar em conta a seleção nas informações de peso adulto (Meyer, 1995; Kaps et al., 1999).

De modo geral, as estimativas de herdabilidade para peso adulto das fêmeas, especialmente aquelas obtidas com medidas repetidas e em modelos trivariados, concordam com as estimadas em populações criadas em regiões temperadas e foram mais altas que aquelas estimadas em populações criadas sob clima tropical. Koots et al. (1994) relataram valor de herdabilidade de peso adulto das matrizes igual a $0,50 \pm 0,02$, média de 25 estudos com bovinos criados em países de clima temperado, enquanto Lôbo et al. (2000) observaram valor de

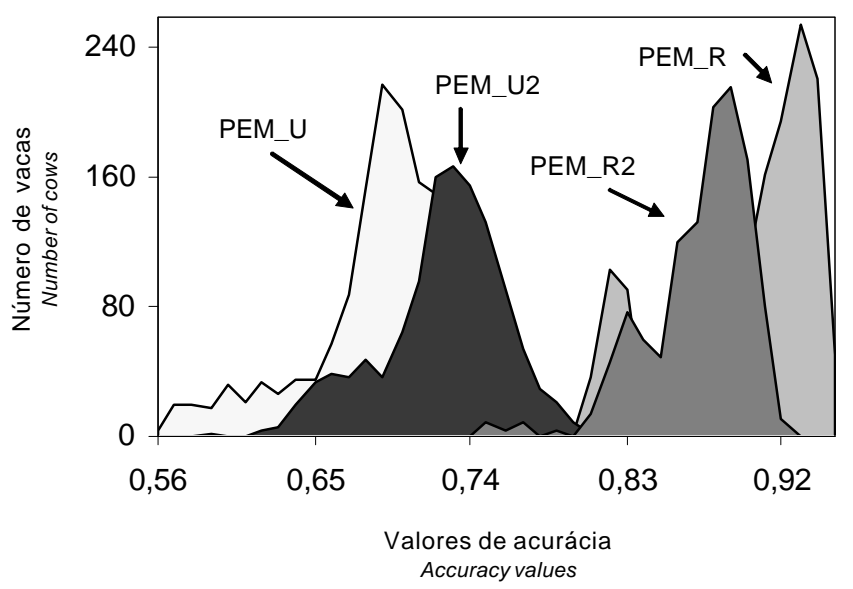

Figura 3 - Distribuição dos valores de acurácia associados aos valores genéticos das vacas com registros, obtidos em análises trivariadas de PEM U, PEM U2, PEM R e PEM R2.

Figure 3 - Distribution of accuracy values associated to breeding values of cows with records, obtained from multiple-trait analysis of PEM_U, PEM_U2, PEM_R and PEM_R2. 
herdabilidade bem inferior $(0,28 \pm 0,05)$, média de cinco estudos com bovinos criados em países tropicais. Em zebuínos, Rosa et al. (2000) e Rosa et al. (2001), com um só registro do peso de fêmeas Nelore, obtiveram valores de herdabilidade iguais a $0,36 \mathrm{e}$

Tabela 4 - Parâmetros genéticos e de ambiente para peso na entrada da monta (PEM), estimados em análises trivariadas

Table 4 - Genetic and environmental parameter estimates for weight at the beginning of the breeding season (PEM), obtained from multiple trait analyses

\begin{tabular}{lllc}
\hline Caracter & $\mathrm{h}^{2}$ & $\mathrm{c}^{2}$ & $\mathrm{t}$ \\
Trait & & & \\
\hline PEM_U & 0,34 & & \\
PEM_U2 & 0,42 & & \\
PEM_R & 0,56 & 0,05 & 0,61 \\
PEM_R2 & 0,57 & 0,15 & 0,72 \\
\hline
\end{tabular}

PEM: peso na entrada da estação de monta; U: considerando somente o último peso disponível para cada vaca; _U2: como_U, excluindo vacas descartadas dos rebanhos antes de 4 anos de idade; _R: considerando todos os registros disponíveis para cada vaca - medidas repetidas; _R2: como_R, excluindo vacas descartadas dos rebanhos antes de 4 anos e registros tomados antes de 4 anos de idade; $h^{2}$, herdabilidade, $c^{2}$, porção da variância total devida ao efeito permanente de ambiente; $t$, repetibilidade.

PEM: weight at the beginning of breeding season;_U: considering only the last record available for each cow; U2: same as U, excluding cows culled from the herds before 4 years of age; $R$ : considering all records available for each cow - repeated records; _R2: same as_R, excluding cows culled from the herds before 4 years and records taken before 4 years of age. $h^{2}$, heritability, $c^{2}$, proportion of phenotypic variance due to permanent environmental effects; $t$, repeatability.
0,26, respectivamente, em um e em vários rebanhos, e Arango \& Plasse (2002), utilizando todos os registros disponíveis do peso de entrada na monta e desmame das matrizes, encontraram valores entre $0,49 \pm 0,10$ e $0,68 \pm 0,07$ para um rebanho experimental de animais Brahman. $\mathrm{O}$ alto valor de herdabilidade obtido no presente estudo pode ser creditado ao fato de os dados serem provenientes somente de um rebanho, que, segundo Rosa et al. (2001), permite que as variações ambientais sejam melhor modeladas, como também do uso de medidas repetidas em modelo multivariado com um caracter anterior à seleção, como ressaltado por Kaps et al. (1999).

As estimativas do ganho genético correlacionado no peso adulto das fêmeas, decorrentes da seleção para peso ao sobreano, em cada rebanho experimental, obtidas do modelo trivariado com PEM_R2, foram iguais a $-0,67 \pm 0,44 \mathrm{~kg} / \mathrm{ano}(\mathrm{p}=0,91), 1,72 \pm 0,33 \mathrm{~kg} / \mathrm{ano}$ $(\mathrm{p}=0,00)$ e $1,51 \pm 0,31 \mathrm{~kg} / \mathrm{ano}(\mathrm{p}=0,00)$, respectivamente, para $\mathrm{NeC}, \mathrm{NeS}$ e NeT. Mercadante et al. (2003a) obtiveram estimativas de mudança genética do peso à seleção de machos e fêmeas, analisados como um somente, iguais a $-0,06 \pm 0,30 \mathrm{~kg} / \mathrm{ano}$ para $\mathrm{NeC}$, $1,74 \pm 0,21 \mathrm{~kg} /$ ano para $\mathrm{NeS}$ e $2,35 \pm 0,21 \mathrm{~kg} / \mathrm{ano}$ para NeT. Estes valores não são diretamente comparáveis, em função da diferença de escala entre o peso à seleção e o PEM. Os valores correspondentes, expressos como porcentagem da média dos animais nascidos durante os quatro

Tabela 5 - Coeficientes de correlação de Spearman entre valores genéticos preditos (VG) para PEM, obtidos de análises multivariadas, de touros dos rebanhos seleção (NeS) e tradicional (NeT), acima da diagonal, e de todos os animais, abaixo da diagonal

Table 5 - Spearman correlation coefficients between predicted breeding values (VG) for weight at the beginning of the breeding season (PEM), obtained from multiple trait analyses for sires only, above the diagonal, and for all the animals, below the diagonal

\begin{tabular}{lcccc}
\hline $\begin{array}{l}\text { Variável } \\
\text { Variable }\end{array}$ & VG_PEM_U & VG_PEM_U2 & VG_PEM_R & VG_PEM_R2 \\
\hline VG_PEM_U & 1,00 & 0,98 & 0,87 & 0,95 \\
VG_PEM_U2 & 0,98 & 1,00 & 0,95 & 0,95 \\
VG_PEM_R & 0,87 & 0,85 & 1,00 & 0,91 \\
VG_PEM_R2 & 0,93 & 0,94 & 0,94 & 1,00
\end{tabular}

VG: valor genético predito; PEM: peso na entrada da estação de monta; _U: considerando somente o último peso disponível para cada vaca; U2: como_U, excluindo vacas descartadas dos rebanhos antes de 4 anos de idade; R: considerando todos os registros disponíveis para cada vaca - medidas repetidas; _R2: como _R, excluindo vacas descartadas dos rebanhos antes de 4 anos e registros tomados antes de 4 anos de idade. VG: predicted breeding value; PEM: weight at the beginning of the breeding season; U: considering only the last record available for each cow; _U2: same as_U, excluding cows culled from the herds before 4 years of age; R: considering all records available for each cow-repeated records; $R 2$ : same as_ $R$, excluding cows culled from the herds before 4 years and records taken before 4 years of age. 
primeiros anos do experimento (1981 a 1984) de cada rebanho, foram $-0,16 \pm 0,11 \%$ /ano, $0,40 \pm 0,08 \% /$ ano e $0,35 \pm 0,07 \%$ /ano para os rebanhos $\mathrm{NeC}, \mathrm{NeS}$ e NeT, enquanto os respectivos valores relatados por Mercadante et al. (2003a) para peso à seleção foram $-0,02 \pm 0,11 \%$ /ano, $0,64 \pm 0,08 \%$ /ano e $0,87 \pm 0,08 \%$ / ano. Em NeS, a resposta correlacionada no peso adulto das fêmeas foi cerca de $62 \%$ da resposta direta no peso à seleção, enquanto no NeT este valor foi menor, cerca de $40 \%$. Pela correlação genética positiva e de média à alta magnitude entre P378 e P550 com PEM_R2 (0,44 e 0,80), esperar-se-iam ganhos genéticos anuais positivos e significativos para os rebanhos selecionados $\mathrm{NeS}$ e NeT e nãosignificativo para NeC. Rosa (1999), citado por Arango \& Plasse (2002), e Arango \& Plasse (2002) relataram mudanças genéticas para peso adulto das fêmeas bem inferiores às obtidas nos rebanhos selecionados $\mathrm{NeS}$ e NeT, iguais a 0,4 e $0,7 \mathrm{~kg} / \mathrm{ano}$, respectivamente.

Apesar de a alta variabilidade genética estimada para PEM indicar a possibilidade de ganho genético via seleção para a modificação do peso adulto das vacas, a correlação com peso ao sobreano é positiva e relativamente alta, e restrição no peso adulto das vacas seria às custas de menores ganhos genéticos no peso ao sobreano. A Figura 4, com os valores genéticos de 133 touros dos rebanhos selecionados

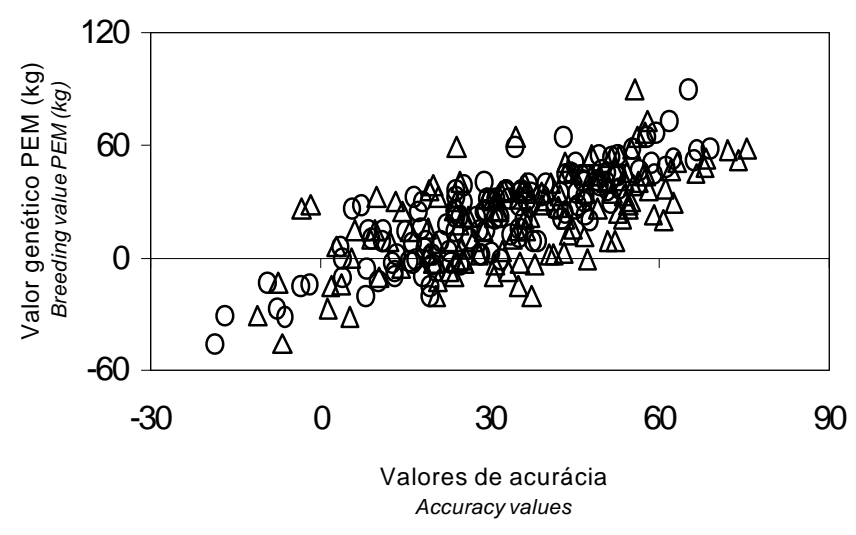

Figura 4 - Valores genéticos dos touros para peso das vacas na entrada da estação de monta (PEM) plotados em relação aos valores genéticos dos touros para peso aos 378 dias (? ) e para peso aos 550 dias (o).

Figure 4 - Sire breeding values of weight of cows at the beginning of breeding season (PEM) plotted against their respective breeding values for P378-day (?) and P550-day (o) weights.
NeS e NeT plotados em relação aos valores genéticos de P378 e P550, mostra que touros com alto valor genético para P378 ou P550 e valor genético negativo para PEM_R praticamente não existem na população estudada, entretanto, é possível encontrar animais com alto valor genético de P378 e P550 e valores genéticos moderados de PEM. Os valores genéticos estão expressos como desvio da população base, ou seja, dos pais dos animais que nasceram de 1978 em diante.

Na comparação empírica de modelos univariados e trivariados utilizando medida única e medidas repetidas do PEM como indicativo do peso adulto das matrizes, o melhor modelo, considerando os componentes de variância e covariância das características, e a acurácia dos valores genéticos preditos, foi o trivariado, com pesos anteriores à seleção, e utilizando medidas repetidas do peso de entrada na monta sem os registros tomados aos 2 e 3 anos de idade. Esta modelagem poderia ser usada atualmente na inclusão do peso adulto das matrizes em avaliações genéticas de reprodutores. Entretanto, deve ser ressaltado que não foram aqui incluídas pelo menos duas abordagens/modelagens possíveis de análise de peso adulto com dados longitudinais: o uso, já bem difundido, de curva não-linear de crescimento e a inclusão em uma análise trivariada do parâmetro A, o que seria uma análise mais parcimoniosa que a de PEM_R, e a análise de regressão aleatória utilizando todos os pesos do animal, da seleção até o descarte. Com relação às curvas não-lineares de crescimento, Van der Werf \& Schaeffer (1997) destacam que uma das desvantagens do método de "dois-passos", a estimação dos parâmetros da curva e a subsequente estimação dos componentes de variância dos parâmetros, é a não utilização das informações dos parentes no primeiro passo, ou seja, na estimação dos parâmetros da curva. Com relação à análise de regressão aleatória, apesar destes modelos serem os mais apropriados para dados longitudinais, funções de covariância genética do momento da seleção até o descarte não estão ainda disponíveis na literatura e a alta demanda em recursos computacionais tem, até o momento, inviabilizado o emprego da metodologia em sistemas de avaliações genéticas que fazem uso de grandes bancos de dados.

\section{Conclusões}

Há evidências de que o emprego de modelos multivariados incluindo registros repetidos e os pesos

R. Bras. Zootec., v.33, n.5, p.1135-1144, 2004 
à seleção de machos e fêmeas seja mais apropriado que a utilização de apenas o último PEM como indicativo do peso adulto.

Altas correlações entre as classificações dos animais foram observadas; dependendo da situação, o último peso da matriz, em análise multivariada, poderia ser utilizado em uma avaliação genética como indicativo do peso adulto, porém, com perda de acurácia dos valores genéticos preditos.

A inclusão dos registros das vacas de 2 e 3 anos de idade na análise de PEM aumenta a acurácia das avaliações nas análises multivariadas, porém com repetibilidade menor e parâmetros genéticos fora do padrão esperado.

O PEM poderia ser incluído na seleção de touros e novilhas de reposição, visando mudança (aumento ou diminuição) ou monitoração para manter peso adulto desejável.

\section{Literatura Citada}

ARANGO, J.; PLASSE, D. Cow weight in a closed Brahman herd. In: WORLD CONGRESS ON GENETICS APPLIED TO LIVESTOCK PRODUCTION, 7., 2002, Montpellier. Proceedings... Montpellier: 2002. CD-ROM.

BOLDMAN, K.G.; KRIESE, L.A.; Van VLECK, L.D. et al. A manual for use for MTDFREML. A set of programs to obtain estimates of variances and covariances. Lincoln: Department of Agriculture; Agricultural Research Service, 2002. 129p.

BULLOCK, K.D.; BERTRAND, J.K.; BENYSHEK, L.L. Genetic and environmental parameters for mature weight and other growth measures in polled Hereford cattle. Journal of Animal Science, v.71, p.1737-1741, 1993.

CHOY, Y.H.; BRINKS, J.S.; BOURDON, R.M. Repeatedmeasure animal models to estimate genetic components of mature weight, hip height, and body condition score. Journal of Animal Science, v.80, p.2071-2077, 2002.

GARNERO, A.V. Modelos não lineares para descrever o crescimento de fêmeas da raça Nelore. Ribeirão Preto: Universidade de São Paulo, 2002. 102p. Tese (Doutorado em Ciências) - Universidade de São Paulo, 2002.

JOHNSON, R.K.; NIELSEN, M.K.; CASEY, D.S. Responses in ovulation rate, embryonal survival, and litter traits in swine to 14 generations of selection to increase litter size. Journal of Animal Science, v.77, p.541-557, 1999.

JOHNSTON, D.J.; CHANDLER, H.; GRASER, H-U. Genetic parameters for cow weight and condition score in Angus, Hereford, and Poll Hereford cattle. Australian Journal of Agricultural Research, v.47, p.1251-1260, 1996.

KAPS, M.; HERRING, W.O.; LAMBERSON, W.R. Genetic and environmental parameters for mature weight in Angus cattle. Journal of Animal Science, v.77, p.569-574, 1999.
KOOTS, K.R.; GIBSON, J.P.; SMITH, C. et al. Analyses of published genetic parameters estimates for beef production traits. 1. Heritability. Animal Breeding Abstracts, v.62, p.309-338, 1994.

LÔBO, R.N.B.; MADALENA, F.E.; VIEIRA, A.R. Average estimates of genetic parameters for beef and dairy cattle in tropical regions. Animal Breeding Abstracts, v.68, p.433-462, 2000.

MERCADANTE; M.E.Z.; PACKER, I.U.; RAZOOK, A.G. et al. Direct and correlated responses to selection for yearling weight on reproductive performance of Nelore cows. Journal of Animal Science, v.81, p.376-384, 2003a.

MERCADANTE, M.E.Z.; RAZOOK, A.G.; CYRILlo, J.N.S.G. et al. Efeito da seleção para crescimento na permanência de vacas Nelore no rebanho até cinco anos de idade. Revista Brasileira de Zootecnia, v.33., n.2, p.344-349, 2004.

MERCADANTE, M.E.Z.; RAZOOK, A.G.; TROVO, J.B.F. et al. Análises alternativas do peso adulto de vacas Nelore em rebanhos experimentais. In: REUNIÃO ANUAL DA SOCIEDADE BRASILEIRA DE ZOOTECNIA, 40., 2003b, Santa Maria. Anais... Santa Maria: Sociedade Brasileira de Zootecnia. CD-ROM.

MEYER, K. Estimates of genetic parameters for mature weight of Australian beef cows and its relationship to early growth and skeletal measures. Livestock Production Science, v.44, p.125-137, 1995.

OLIVEIRA, H.N.; LÔBO, R.B.; PEREIRA, C.S. Comparação de modelos não lineares para descrever o crescimento de fêmeas da raça Guzerá. Pesquisa Agropecuária Brasileira, v.35, p.1843-1851, 2000.

RAZOOK, A.G.; FIGUEIREDO, L.A.; BONILHA NETO, L.M. et al. Selection for yearling weight in Nelore and Guzera zebu breeds: selection applied and response in 15 years of progeny. In: WORLD CONGRESS ON GENETICS APPLIED TO LIVESTOCK PRODUCTION, 6., 1998, Armidale. Proceedings... Armidale: 1998. CD-ROM.

ROSA, A.N.; LÔBO, R.B.; OLIVEIRA, H.N. et al. Peso adulto de matrizes em rebanhos de seleção da raça Nelore no Brasil. Revista Brasileira de Zootecnia, v.30, p.1027-1036, 2001.

ROSA, A.N.; LÔBO, R.B.; OLIVEIRA, H.N. et al. Variabilidade genética do peso adulto de matrizes em um rebanho Nelore do Estado de São Paulo. Revista Brasileira de Zootecnia, v.29, p.1706-1711, 2000.

STATISTICAL ANALYSES SYSTEM - SAS.SAS/STAT 2000: version 8. Cary: 2000.

SILVA, A.M.; ALENCAR, M.M.; FREITAS, A.R. et al. Herdabilidades e correlações genéticas para peso e perímetro escrotal de machos e características reprodutivas e de crescimento de fêmeas, na raça Canchim. Revista Brasileira de Zootecnia, v.29, p.2223-2230, 2000.

Van der WERF, J.; SCHAEFFER, L. Random regression in animal breeding. Course Notes. CGIL Guelph, 25-28 june, 1997.

Recebido em: 03/06/03 Aceito em: 11/12/03 